\title{
Diseño, Implementación y Evaluación del Programa Transversal de Alfabetización Académica Lector-es
}

\author{
Nelly E. Pardo-Espejo(1)* y Juan D. D. Villanueva-Roa ${ }^{(2)}$ \\ (1) Facultad de Estudios a Distancia, Escuela de Ciencias Humanísticas y Educación, Universidad Pedagógica y \\ Tecnológica de Colombia,Tunja, Boyacá, Colombia. (e-mail: nelly.pardo@uptc.edu.co) \\ (2) Facultad de Ciencias de la Educación, Departamento de Didáctica de la Lengua y Literatura, \\ Universidad de Granada, Granada, España. (e-mail: jvillanueva@ugr.es) \\ *Autor a quien deba ser dirigida la correspondencia
}

Recibido Feb. 7, 2019; Aceptado Abr. 3, 2019; Versión final May. 27, 2019, Publicado Dic. 2019

\begin{abstract}
Resumen
Este artículo describe el diseño, implementación y evaluación del programa transversal de alfabetización académica Lector-es en estudiantes de formación inicial docente en la modalidad de educación a distancia de la Licenciatura en Educación Básica, con énfasis en Matemáticas, Humanidades y Lengua Castellana. El estudio, realizado en la Universidad Pedagógica y Tecnológica de Colombia, presenta un enfoque mixto, de tipo investigación evaluativa sustentada bajo el modelo de evaluación de programas de Pérez Juste. Los resultados ponen de manifiesto que el programa Lector-es responde a las necesidades de los destinatarios y se adecua a los criterios de pertinencia, calidad intrínseca y viabilidad. En la evaluación final, el programa ha presentado un alto grado de eficacia, eficiencia y efectividad. En conclusión, el programa promueve la enseñanza de la lectura como eje transversal del curriculum, la apropiación de los contenidos de las asignaturas y la participación de actividades propias del docente de educación básica.
\end{abstract}

Palabras clave: lectura; programa de alfabetización; evaluación; educación a distancia; formación docente

\section{Design, Implementation and Evaluation of the Transversal Program of Academic Literacy Lector-es}

\begin{abstract}
This article describes the design, implementation and evaluation of the transversal program of academic literacy Lector-es in university students of teacher training in the distance education program of the bachelor in elementary education, with emphasis on mathematics, humanities and Spanish language. The study, done at the Universidad Pedagógica y Tecnológica de Colombia, has a mixed approach of evaluative research type, based on the educational program evaluation known as Pérez Juste model. The results show that the program Lector-es responds to the needs that the participants have and it fulfills the criteria of relevance, intrinsic quality and viability. During the final evaluation, the program showed a high degree of efficacy, efficiency and effectiveness. In conclusion, the program promotes teaching of reading as a transversal axis of the curriculum, appropriation of the contents of the subjects, and taking part in activities that are intrinsic to the basic education teacher.
\end{abstract}




\section{INTRODUCCIÓN}

Durante las últimas décadas, la lectura y la escritura han sido objeto de numerosos estudios en la educación superior, los iniciadores en investigar sobre las prácticas de la lectura y la escritura fueron las universidades australianas y norteamericanas. Entre los estudios anglosajones sobresalen Ivanic y Lea (2006); Lea y Street (2008); Newell y Winograd (1989), estos ayudaron a los estudiantes a leer y a escribir en las universidades; de esta forma, surge el movimiento "Escribir a través del currículum" (Bazerman et al., 2005), con el propósito de integrar la escritura en las diferentes disciplinas. Referente a las investigaciones australianas se encuentran las de Biggs (1999); Chalmers y Fuller (1996); Radloff (1998); Skillen et al., (1998), quienes integraron los conceptos de una disciplina con la enseñanza de estrategias de estudio para aprender esos contenidos a través de la lectura y la escritura como prácticas para acceder, participar en los campos discursivos disciplinares y asumir posición crítica en la producción del conocimiento.

A nivel de Latinoamérica, Argentina fue uno de los países iniciadores en investigaciones sobre prácticas de la lectura y la escritura en la educación universitaria, estas en su mayoría fueron estudios diagnósticos, que tendían a identificar las dificultades que presentaban los estudiantes en la lectura y escritura, lo que conllevó a la creación de talleres y cursos de lenguaje para estudiantes de primer semestre, pero desligados de las asignaturas e insuficientes, dado que al ingresar los estudiantes se enfrentaban a nuevas formas discursivas, en las cuales se les pedía leer y escribir de una forma diferente y con diferente bibliografía (Carlino, 2013). Por tanto, Carlino (2018); Cartolari et al., (2013); Marinkovich, et al., (2016) y Waigandt, et al., (2016) realizan estudios sobre la enseñanza de la lectura y escritura como prácticas propias de sus disciplinas en la formación universitaria. De esta manera, se han ido creando programas de alfabetización académica a lo largo del plan de estudios en las carreras universitarias como política institucional (Moyano, 2018; Moyano y Giudice, 2016).

En los últimos años, el término de alfabetización académica, se utiliza para referirse al proceso de integrar la enseñanza de la lectura y la escritura en las disciplinas. Carlino (2003) lo definió como "el conjunto de nociones y estrategias necesarias para participar en la cultura discursiva de las disciplinas así como en las actividades de producción y análisis de textos requeridas para aprender en la universidad". Diez años después, la autora aclara que "alfabetizar académicamente equivale a ayudar a participar en prácticas discursivas contextualizadas, lo cual es distinto de hacer ejercitar habilidades que las fragmentan y desvirtúan" (Carlino, 2013). Por consiguiente, la alfabetización académica no está relacionada con los procesos de enseñanza y aprendizaje inicial de la lectura y la escritura, sino como proceso en desarrollo a lo largo de toda la formación universitaria (Sánchez Upegui, 2016). Por tanto, la alfabetización se logra a través del currículo y al interior de las disciplinas, definidas estas como "espacios discursivos y retóricos, tanto como conceptual" (Bogel y Hjortshoj, 1984), lo que significa que aprender una disciplina no consiste sólo en adquirir su sistema de conceptos y métodos, sino en manejar sus modos de leer y escribir característicos y en usar sus géneros discursivos específicos (Parodi, et al., 2010). En este sentido, el objetivo de esta alfabetización académica es desarrollar en los estudiantes la capacidad de comunicarse adecuadamente en los diversos contextos en los que se manejan, utilizando los recursos discursivos propios de cada disciplina particular con el fin de generar conocimientos en cada una de ellas (Castelló, 2014). Por ello, el hacerse cargo de la enseñanza de la lectura y la escritura de los estudiantes es tarea compartida de los docentes de las diferentes asignaturas, profesores de lenguaje, estudiantes e instituciones, dado que esto contribuye al aprendizaje de los conceptos teóricos de las disciplinas, a la formación profesional y a la participación de una cultura académica universitaria.

En Colombia hacia el año 2003, la Asociación Colombiana de Universidades ASCUN, en colaboración con el Instituto Colombiano para la Evaluación de la Educación ICFES contribuyeron en gran medida para que los procesos de lectura y escritura fueran objeto de análisis y de estudio, dado que por este tiempo, se aplicó por primera vez en el país, una prueba de competencias genéricas a los futuros profesionales del país denominada Exámenes de Calidad de Educación Superior ECAES, ahora (Saber Pro), en la que se demostró que "Ios universitarios no saben leer ni escribir, dificultades que se observan en la mala redacción y en la poca capacidad de análisis y comprensión lectora" (González y Vega, 2013). Por tanto, se produjo una fuerte discusión en torno a la lectura y escritura, sus prácticas y formas de enseñanza, originándose investigaciones, experiencias y políticas educativas para el fortalecimiento de los procesos de lectura y escritura en las instituciones educativas superiores.

Entre las investigaciones sobresalen la de Salazar et al. (2015), quienes durante los años 2008-2010 realizan un estudio explorativo, en la que participaron diez universidades colombianas con 34 experiencias de lectura y escritura, de las cuales se pudo establecer que en su mayoría se ocupan de la lectura y la escritura como competencias generales y no como prácticas socioculturales, y al diseño de cursos remediales. Por esta misma época, Pérez y Rincón (2013) desarrollan una investigación financiada por el Departamento Administrativo de Ciencia, Tecnología e Innovación, COLCIENCIAS en programas de pregrado presenciales de 17 universidades colombianas entre los años 2009 y 2011, en esta se determinó que los estudiantes universitarios presentan dificultades en la comprensión y producción textual. Luego durante los años del 2011 
al 2015, González, et al. (2015) investigan sobre la formación inicial de la lectura y escritura en doce universidades en la modalidad presencial y una a distancia, comprobándose la ausencia de la lectura para aprender, dado que esta se utiliza para escribir y/o para discutir.

De tal manera, que las problemáticas mencionadas anteriormente, poco se han estudiado en las carreras de modalidad de educación a distancia, máxime cuando esta modalidad exige a los estudiantes un alto grado de comprensión e interpretación textual en sus actividades académicas, con el propósito de desarrollar sus capacidades para entrenarse de forma independiente y autónoma. De otra parte, la Universidad Pedagógica y Tecnológica de Colombia UPTC en su estructura curricular en el área general, propone en todos los programas académicos presenciales y a distancia una asignatura obligatoria "Competencias Comunicativas" en el primer semestre, orientada por profesores especializados en lenguaje. Esta pretende desarrollar las habilidades comunicativas constituyéndose como una forma para enseñar la lectura y la escritura como técnicas aplicables a todos los textos, propósitos y contextos, evidenciándose la lectura y la escritura como habilidades generales que se logran de una vez y para siempre, y no como proceso en desarrollo. Por esta razón, los docentes del área disciplinar y de profundización de la Licenciatura en Educación Básica tienen la idea que al ingresar los estudiantes a la universidad vienen formados en la lectura y la escritura; por consiguiente, su enseñanza no es tarea de ellos.

Pese a que los estudiantes cursan esta asignatura, los resultados no han sido satisfactorios, ya que los estudiantes continúan con grandes dificultades en el abordaje de los géneros discursivos propuestos en las asignaturas. Por lo que se verifica que estos cursos son insuficientes, dado que los estudiantes se encuentran con variedad de géneros, en los cuales se les exige leer de una forma diferente a lo que estaban acostumbrados en etapas educativas previas. Esto conlleva a cuestionar la efectividad de estos cursos iniciales y considerar ajustes necesarios para la solución de esta problemática, lo que significa pensar de manera transversal la lectura como un proceso continuo que se dé a lo largo de la carrera y no en un solo curso de 16 semanas que desarrolle las competencias necesarias para apropiación del conocimiento de las asignaturas y la participación de una cultura letrada, con el propósito de preparar a los estudiantes para un mejor desempeño en la universidad, así como para su futura actuación como profesionales (Poblete et al., 2017).

Por lo anterior, surge la necesidad de diseñar, implementar y evaluar el programa transversal de alfabetización académica "Lector-es", proceso realizado durante los años de 2016-2018, en la Facultad de Estudios a Distancia FESAD de la Universidad Pedagógica y Tecnológica de Colombia UPTC como oportunidad para mejorar y fortalecer los procesos de enseñanza y aprendizaje de la lectura, situándolos en contextos de las asignaturas del área disciplinar y profundización, entendida como "saberes, competencias y prácticas que determinan el perfil estricto y específico de un Programa Académico que define una profesión y responde a los campos del saber de la respectiva disciplina" (Acuerdo 050, Art. $6^{\circ}, 2009$ ). Además como medio para desarrollar la autonomía del estudiante en los procesos de lectura, sobre todo cuando la modalidad de educación a distancia se basa en el aprendizaje auto dirigido; asimismo, contribuir con la ejecución de la Cátedra UNESCO, debido a que la lectura y la escritura son para esta organización criterios claves para mejorar la calidad de la educación y la equidad; del mismo modo, el rendimiento académico e investigativo que conlleve a una adecuada actuación social, científica y laboral de los estudiantes latinoamericanos. Por último, favorecer el desarrollo de una sociedad más crítica y comprometida a través de la "formación de lectores competentes y críticos, capaces de acceder por sí mismos a los textos y estar preparados para realizar los aprendizajes necesarios para optimizar su desarrollo personal, social y profesional" (Yubero y Larrañaga, 2015).

\section{Descripción del programa}

El programa transversal de Alfabetización Académica "Lector-es", ha sido diseñado para implementarlo en la Licenciatura en Educación Básica, con énfasis en Matemáticas, Humanidades y Lengua Castellana perteneciente a la Facultad de Estudios a Distancia de la Universidad Pedagógica y Tecnológica de Colombia, Institución Educativa de carácter pública, creada en 1953 con sede y domicilio principal en Tunja, BoyacáColombia y con sedes seccionales en Duitama, Sogamoso y Chiquinquirá, además con Centros Regionales de Educación a Distancia CREAD. El programa "Lector-es" está dirigido a estudiantes de formación inicial docente de la modalidad de educación a distancia, definida como "conjunto de relaciones pedagógicas entre estudiantes, docentes e institución, basadas o apoyadas en el uso de tecnologías para el desarrollo sistémico de procesos formativos de calidad" (Salazar y Melo, 2013, p.102), con metodología B-learning en la que se combina una metodología de enseñanza y aprendizaje presencial, con una metodología de enseñanza y aprendizaje virtual, donde "se disponen espacios (clases tradicionales y virtuales), tiempos (presenciales y no presenciales), recursos (analógicos y digitales), donde los protagonistas modifican sus roles en los procesos de enseñanza/aprendizaje, y donde los cambios también afectan, de manera ineludible, a los modelos organizativos" (Cabero y Llorente, 2008). 
En este sentido, el programa "Lector-es", se fundamenta en la pedagogía constructivista, desde la concepción del sujeto como participante activo de su proceso de aprendizaje, en el que se promueve al aprendizaje autónomo y autorregulado, en donde se concibe al estudiante como protagonista y gestor de su aprendizaje en interacción con el tutor, y al docente como facilitador y acompañante en la formación del estudiante como ser integral y capaz de participar en actividades discursivas propias de las asignaturas del área disciplinar y de profundización para ser miembro activo de una cultura académica. Al considerar el estudiante como constructor de su propio conocimiento, el programa dota al estudiante de estrategias cognitivas, metacognitivas y socio afectivas, las cuales le permiten al participante comprender y desarrollar las actividades; planificar, monitorear y evaluar su aprendizaje e interactuar con sus compañeros para construir y transformar el conocimiento. Asimismo, el programa brinda al estudiante espacios didácticos para que el encuentro del estudiante con la información se haga de una manera significativa, a través de estrategias organizativas como el estudio independiente, estudio en pequeño y gran grupo, Del mismo modo, ofrece medios y recursos para la mediación pedagógica como medios tradicionales entre ellos guías de lectura, protocolos, libros etc. medios tecnológicos como la plataforma virtual moodle, medios de interacción mediante la comunicación asincrónica y sincrónica, y recursos multimediales sustentados en el uso de los videos, audios, presentaciones digitales, entre otros.

El programa consta de cinco (5) secuencias didácticas y 15 sesiones, cada una de ellas integra la lectura con el aprendizaje al interior de los contenidos de las asignaturas del área disciplinar y profundización "Fundamentos y didácticas de las artes plásticas", "Fundamentos y Didácticas de la Educación Física", "Lengua Castellana para la Educación Básica Primaria", "Profundización en el área del Inglés para la Educación Básica" y Profundización en el área de las matemáticas para la Educación Básica. Asimismo, cuenta con guías de lectura estructuradas bajo los tres momentos de la lectura: antes, durante y después, a través de la lectura de textos académicos y científicos, orientados y centrados a fortalecer la lectura literal, inferencial y critico intertextual, del mismo modo, a desarrollar habilidades de orden superior, a aprender un contenido, y por último a participar en actividades especializadas de la formación docente.

El programa "Lector-es" tiene como propósito integrar la lectura en el currículo como práctica de apropiación del conocimiento de las disciplinas y la participación en una cultura académica universitaria (Bogel y Hjortshoj, 1984; Rusell, 2013); de la misma manera, favorecer la comprensión lectora a partir de actividades de lectura en las disciplinas que integren la teoría con la práctica; formar lectores autorregulados, quienes participan de actividades propias de un docente de Educación Básica, de forma consciente, crítica y autónoma; generar el espacio curricular e interdisciplinar necesario para la enseñanza de la lectura académica; diseñar y aplicar secuencias didácticas que integren el proceso lector con los contenidos específicos de las asignaturas del área disciplinar y profundización, actualizar a los docentes a fin de asumir con responsabilidad los procesos de la lectura de sus estudiantes en sus asignaturas. Finalmente, impulsar a docentes y estudiantes a presentar proyectos, ponencias y artículos tanto en revistas y congresos como producto de procesos de lectura de sus asignaturas.

La alfabetización académica no estuvo pensada como una propuesta para una asignatura específica, tampoco como asunto remedial, sino como un programa transversal que consiste en "abrir las puertas a los alumnos a las especificidades de cada disciplina, siendo imprescindible para ello acompañarles en la comprensión del lenguaje científico y de los textos académicos, por lo que la lectura sería la base de este aprendizaje" (Yubero y Larrañaga, 2015, p. 719). Por esta razón, el programa se fundamenta en el enfoque de la enseñanza en contexto (Carlino, 2013), lo que significa incluir la enseñanza de la lectura de los géneros discursivos asociados al campo académico y al profesional (Calle Arango, 2018; Sánchez Upegui, 2016) en las asignaturas del área de formación disciplinar y de profundización. Igualmente, se apoya en el principio de la lectura en función epistémica como actividad estratégica para pensar y aprender (Colombo y Prior, 2016; Solé, 2012) y como práctica sociocultural (Santos, 2016).

En este sentido, el programa no solo se ocupa de la enseñanza de la lectura y del aprendizaje de los contenidos, sino también de la participación de los estudiantes en "actividades similares a las que realizan los especialistas de cada disciplina, para que logren un aprendizaje profundo de los conocimientos disciplinares para que puedan, a su vez, transformar y producir nuevo conocimiento" (Moje, 2007 y 2008, citado en LópezBonilla, 2013, p. 388). Lo anterior, para contribuir con el fortalecimiento de una de las competencias básicas y fundamentales del maestro como es la de "enseñar, la cual hace referencia a "comprender, formular y usar la didáctica de las disciplinas con el propósito de favorecer los aprendizajes de los estudiantes y con ellos el desarrollo de las competencias propias del perfil profesional" (MEN, 2014). Es aquí donde el estudiante tiene la oportunidad de aprender un contenido, con el cual podrá construirlo y aplicarlo a un nuevo contexto a través de la lectura como medio fundamental para asimilar y transformar el contenido con el propósito de aprender de una forma significativa y de participar activamente en una cultura académica propia de su especialidad. Es así como los docentes especialistas en lenguaje y los de las disciplinas de la licenciatura son los directos responsables de los procesos de lectura de los estudiantes en formación inicial docente para garantizar el aprendizaje de los contenidos de las asignaturas implicadas. 


\section{METODOLOGÍA}

Este estudio presenta un enfoque mixto, definido como la combinación sistemática de datos cualitativos y cuantitativos en una misma investigación, con diseño de investigación evaluativa referida al proceso sistemático, riguroso y controlado de recogida de y análisis de información fiable y válida, orientado a valorar la calidad y logros como base para tomar decisiones de mejora de un programa educativo (Pérez Juste, 2014). De modo que, para determinar la validez del programa transversal de alfabetización académica "Lector-es", se seleccionó el modelo de este mismo autor. Es así como la tabla 1 explica los momentos cruciales para evaluar un programa educativo: evaluación inicial del diseño del programa, evaluación procesual de la implementación y evaluación final de los resultados. De otra parte, el estudio también presenta un diseño cuasi experimental con medidas de pre-test y pos-test, con dos grupos (experimental y control).

Tabla 1: Modelo de evaluación de programas educativos (Pérez Juste, 2014)

\begin{tabular}{|c|c|c|}
\hline Momentos & Dimensiones & Objeto \\
\hline \multirow{3}{*}{$\begin{array}{l}\text { Evaluación inicial del diseño } \\
\text { del programa }\end{array}$} & Adecuación y Pertinencia & $\begin{array}{l}\text { Destinatarios } \\
\text { Contexto }\end{array}$ \\
\hline & $\begin{array}{c}\text { Calidad Intrínseca del } \\
\text { programa }\end{array}$ & $\begin{array}{l}\text { Objetivos } \\
\text { Metodología } \\
\text { Contenidos } \\
\text { Actividades } \\
\text { Recursos } \\
\text { Evaluación }\end{array}$ \\
\hline & $\begin{array}{l}\text { Adecuación a las } \\
\text { circunstancias }\end{array}$ & Viabilidad \\
\hline \multirow{2}{*}{$\begin{array}{c}\text { Evaluación Procesual de la } \\
\text { implementación del } \\
\text { programa }\end{array}$} & $\begin{array}{l}\text { Puesta en marcha del } \\
\text { programa }\end{array}$ & \multirow{2}{*}{$\begin{array}{c}\text { Cobertura } \\
\text { Temporalización } \\
\text { Actividades } \\
\text { Recursos }\end{array}$} \\
\hline & Marco de aplicación & \\
\hline \multirow{2}{*}{$\begin{array}{l}\text { Evaluación Final de los } \\
\text { resultados de la aplicación } \\
\text { del programa }\end{array}$} & Logros & Constatación \\
\hline & Valoración & Criterios \\
\hline
\end{tabular}

En este sentido, los instrumentos fueron seleccionados de acuerdo con las fases de evaluación de programas educativos. Dado que, "las necesidades son un primer elemento clave a la hora de diseñar un programa educativo" (Pérez Juste, 2014, p.166), se aplicó un cuestionario tipo encuesta (Pérez y Rincón, 2013) y un cuestionario (Mora Venegas, 2017), para identificar las necesidades en las prácticas de lectura de los estudiantes y las prácticas de enseñanza de la misma en los profesores tutores. Relacionado con la evaluación inicial del programa, se diseñó y se aplicó una escala de estimación ad hoc a un grupo de expertos externos para evaluar el diseño del programa. Vale la pena aclarar que este instrumento fue objeto de validez de contenido por juicio de expertos y mediante el coeficiente de Aiken V, asimismo para determinar su fiabilidad, se estableció el coeficiente del alfa de crombach a través del software SPSS versión 20 , obteniéndose el análisis estadístico de alfa de Crombach ( $\alpha$ ) y fiabilidad de $\alpha=0,87$ indicando que el $87 \%$ de la escala es fiable para evaluar el diseño, implementación y evaluación de los resultados del programa. La escala de estimación estuvo constituida por tres secciones con un total de 57 preguntas distribuidas así: 37 preguntas para evaluar el diseño del programa, 12 para la implementación del mismo y 8 para los resultados de la aplicación del programa, con 5 posibles respuestas ( $0=$ nulo; $1=$ bajo; $2=$ medio; $3=$ alto y $4=$ =muy alto) para definir el grado de intensidad de cada pregunta.

En la fase de evaluación procesual de la implementación del programa, se diseñó y se administró un pre-test ad hoc que luego se aplicó como pos-test para identificar el nivel inicial y final de la comprensión literal, inferencial y critico-intertextual. Para la construcción del pre-test, se elaboró una versión inicial de 24 preguntas, con base en dos textos continuos (abstract y artículo de un estudio investigativo), y textos discontinuos (gráfica de barras estadísticas, gráfica lineal estadística de estudios investigativos y una pintura de Frida Kahlo). Para la validez de la prueba de contenido, se contactaron a tres expertos cuyos perfiles correspondieron a profesiones relacionados con la pedagogía en lenguas, lingüística y semiótica, y un profesional psicólogo con conocimiento de metodología de la investigación, quienes evaluaron y validaron las preguntas. Posterior a la evaluación de los jueces, se procedió a realizar el análisis de la información arrojada por ellos a través del Coeficiente de Validez de Aiken (V), verificándose en un $75 \%$ fiable el instrumento, luego se procedió a efectuar la prueba piloto. De otra parte, para establecer la estabilidad de una aplicación a otra, se aplicó un re-test y se verificaron las correlaciones de Pearson, las cuales evidenciaron puntuaciones correlacionadas y significativas, determinándose así la estabilidad en las respuestas de las dos aplicaciones, 
además la concurrencia y asociación entre preguntas respuestas, independientemente de la población a la que se aplicó el cuestionario. Finalmente, la salida del SPSS establece que el $\alpha=0,708$ indicando la fiabilidad del instrumento de $70 \%$, y $\mathrm{V}=75 \%$ para medir los niveles de comprensión lectora. Por tanto, se obtuvo la prueba final de 14 preguntas de selección múltiple con única respuesta: 4 para la comprensión lectora literal, 5 para la inferencial y 5 para el crítico intertextual. De otra parte, se administró la segunda sección de la escala de estimación a profesores para evaluar la implementación del programa. Por último, en la fase de evaluación final de los resultados de la aplicación del programa, se aplicó a profesores tutores la tercera sección de la escala de estimación y a estudiantes el pos-test, y el cuestionario de satisfacción.

La muestra del presente estudio fue no probabilística por conveniencia y estuvo conformada por un total de 150 estudiantes del séptimo, octavo y noveno semestre de la Licenciatura en Educación Básica, con énfasis en Matemáticas Humanidades y Lengua Castellana de la UPTC, con edades entre 21 y 35 años, en su mayoría habitan en zonas urbanas y rurales cercanos a la sede central de Tunja y a los Centros Regionales de Educación a Distancia CREAD, optaron por estudiar en la modalidad de educación a distancia por las ventajas que esta tiene respecto a los tiempos y espacios de aprendizaje, dado que un gran número de ellos son docentes en ejercicio en Instituciones Educativas de zonas rurales. Participaron estudiantes de las sedes de las ciudades de Tunja $(n=104)$, Duitama $(n=14)$, Chiquinquirá $(n=14)$ y Bogotá $(n=18)$, el $85.1 \%$ eran mujeres y el $13.8 \%$ eran hombres, quienes cumplían con los requisitos de inclusión: estudiantes de formación inicial docente en la modalidad de educación a distancia de la UPTC del $7^{\circ}, 8^{\circ}$ y $9^{\circ}$ semestre. Los participantes se distribuyeron en dos grupos, el grupo experimental con 75 estudiantes (50\% de toda la muestra) y el grupo control con 75 sujetos (50\% de toda la muestra), observándose una proporción equivalente. Ambos grupos han seguido una misma programación curricular, si bien el grupo experimental al interior de las asignaturas participan en el programa transversal de alfabetización académica "Lector-es". Igualmente, participaron 6 profesores ocasionales de tiempo completo, con experiencia en las áreas de convocatoria, con criterios de inclusión: profesor tutor de inglés, matemáticas, artes plásticas, educación física o lengua castellana de la UPTC y con experiencia en educación a distancia y virtual.

\section{RESULTADOS}

A continuación se presentan los resultados del programa "Lector-es" de acuerdo con las fases de evaluación de programas educativos propuestas por Pérez Juste (2014), evaluación inicial del diseño del programa, evaluación procesual de la implementación y evaluación final de los resultados, incluyendo la detección de las necesidades.

\section{Fase 1. Evaluación de las necesidades frente a las prácticas de lectura}

De acuerdo con los resultados obtenidos de la encuesta aplicada a estudiantes en formación docente de la Licenciatura en Educación Básica con énfasis en Matemáticas, Humanidades y lengua castellana, se determinó que entre los porcentajes más altos respecto a la experiencia de los estudiantes como lectores en las actividades académicas, el $48,3 \%$ lee para la asignatura; seguido el $17,5 \%$ para un grupo de estudio y el 9.4\% para participar en evento académico. Referente a los tipos de documentos leídos sobresalen los materiales elaborados por el profesor con el $16 \%$, seguido las páginas web con $14,6 \%$ y apuntes de clase con $14,1 \%$ y en bajo porcentaje los informes de investigación con $5,0 \%$ y los artículos científicos con $4,8 \%$. Relacionado con los propósitos de lectura de las actividades académicas en los estudiantes en formación docente, las frecuencias más altas fueron la de responder una evaluación escrita con 19,8\%, continua el de realizar una exposición con 19,1\% y luego el de responder a una evaluación oral con el 14,4\%. Referente a la asignatura en la que vivieron los estudiantes la mejor experiencia significativa de lectura, es de resaltar que el $17 \%$ de los estudiantes no respondieron.

Respecto a las prácticas de enseñanza de la lectura de los profesores tutores de la Licenciatura en Educación Básica con énfasis en Matemáticas, Humanidades y Lengua Castellana, se estableció que entre las prácticas de enseñanza de la lectura, el $21 \%$ de los profesores solicita a sus estudiantes leer materiales elaborados por ellos, el $18 \%$ las páginas de internet, $14 \%$ artículos científicos y en bajas frecuencias los informes de investigación con $1 \%$ y las publicaciones en revistas con $4 \%$. Entre los propósitos de lectura, el $20 \%$ de los docentes solicita a sus estudiantes leer para exponer y sustentar un tema. De otra parte, el $39 \%$ de los docentes cree que para mejorar su forma de orientar la lectura en clase es a través de la aplicación de diferentes técnicas de lectura.

\section{Fase 2. Evaluación inicial del diseño del programa}

Con base en la identificación de las necesidades, se procedió a diseñar el programa transversal de alfabetización académica "Lector-es" para luego evaluar su diseño. Para ello, se eligieron a cinco expertos externos con formación académica y experiencia en áreas de pedagogía, lingüística y en ambientes de 
educación a distancia en el nivel de educación superior. Cada uno de los expertos recibió un documento donde se describía de forma detallada el programa y la primera sección de la escala de estimación que estuvo constituida por 37 preguntas para evaluar las dimensiones de adecuación y pertinencia, calidad intrínseca del programa y adecuación de las circunstancias. Respecto a la dimensión sobre adecuación y pertinencia del programa referida a los destinatarios y al contexto, el grado predominante valorado por los expertos fue de muy alto. La dimensión de calidad intrínseca del programa sobre los objetivos, contenidos, recursos, actividades y evaluación, el grado de cumplimiento estuvo entre alto y muy alto, y la metodología en muy alto. Por último, la dimensión de adecuación de las circunstancias, lo concerniente a la viabilidad, el grado de cumplimiento estuvo entre muy alto y alto.

\section{Fase 3. Evaluación procesual de la implementación del programa}

Una vez dada la viabilidad del programa, se procede a su implementación y a la vez su evaluación. De ello, se obtuvieron los resultados relacionados con el nivel inicial de comprensión lectora y la evaluación de la puesta en marcha y el marco de aplicación del programa, los cuales se presentan a continuación:

\section{Nivel inicial de comprensión lectora}

Una vez se empieza a implementar el programa "Lector-es", se identificó el estado inicial de los niveles de comprensión lectora de los estudiantes de formación docente mediante la aplicación del pre-test. Es así como en la tabla 2, se observa que hay una mínima diferencia entre la media aritmética alcanzada por el grupo experimental frente al grupo control en los diferentes niveles abordados en la prueba. El mayor porcentaje de aciertos en ambos grupos se evidencia en el nivel de comprensión literal, con un nivel de desempeño medio, y para el inferencial y crítico intertextual con un nivel bajo. Esto corrobora los resultados obtenidos en estudios de Durango (2017).

Tabla 2. Media aritmética de respuestas correctas por grupo del pre-test en los niveles de comprensión de lectura

\begin{tabular}{|c|l|r|r|r|}
\hline \multirow{1}{*}{ Grupo } & Estadísticos & Nivel Literal & Nivel Inferencial & Nivel Crítico Intertextual \\
\hline \multirow{5}{*}{ Experimental } & Media & 3,11 & 2,52 & 1,92 \\
\cline { 2 - 5 } & Muestra & 75 & 75 & 75 \\
\cline { 2 - 5 } & $\begin{array}{l}\text { Desviación } \\
\text { Estándar }\end{array}$ & 0,924 & 1,005 & 1,160 \\
\hline \multirow{5}{*}{ Control } & Media & 3,12 & 2,51 & 1,87 \\
\cline { 2 - 5 } & Muestra & 75 & 75 & 75 \\
\cline { 2 - 5 } & Desviación & 1,013 & 1,070 & 1,119 \\
\hline \multirow{5}{*}{ Estándar } & Media & 3,11 & 2,51 & 1,89 \\
\cline { 2 - 5 } & Muestra & 150 & 150 & 1,136 \\
\cline { 2 - 5 } & Desviación & 0,966 & 1,035 & \\
\cline { 2 - 5 } & Estándar & & & \\
\hline
\end{tabular}

\section{Evaluación de la puesta en marcha y el marco de aplicación del programa}

Para verificar si la planificación del programa de alfabetización académica "Lector-es" se estaba desarrollando tal como estaba previsto, durante su implementación se aplicó la segunda sección de la escala de estimación a los profesores tutores implicados en el programa "Lector-es". Esta estuvo constituida por 12 preguntas para evaluar las dimensiones de la puesta en marcha y el marco de aplicación del programa. En cuanto a la dimensión sobre la puesta en marcha del programa, se contempló la cobertura, temporalización, actividades y recursos; por tanto, el $0,23 \%$ de los profesores implicados la valoraron como alto y el $0,77 \%$ muy alto. En cuanto a la dimensión de la aplicación del programa referida a la coherencia y al clima organizacional, su grado de cumplimiento estuvo en muy alto con $7 \%$, alto con $0,3 \%$ y medio con $2,67 \%$.

\section{Fase 4. Evaluación final de los resultados de la aplicación del programa}

Terminado el programa, se procedió a realizar la evaluación final de los resultados del programa; por consiguiente, estos se obtuvieron de los resultados del pos-test, de la aplicación del programa y del grado de satisfacción de los participantes del programa, tal como se expone en los siguientes apartados. 


\section{Nivel final de comprensión lectora}

En cuanto al estado final de los niveles de comprensión lectora del pos-test, la tabla 3 muestra que el desempeño de los estudiantes del grupo experimental mejoró notablemente, encontrando que el promedio de aciertos en los diferentes niveles de comprensión lectora está por encima del $90 \%$ de las preguntas enunciadas en el test. Asimismo, se evidencia que al realizar la comparación con el pre-test, se comprueba que el estudio culmina con un nivel de desempeño alto en cada uno de los niveles de comprensión lectora del grupo experimental.

Tabla 3. Media aritmética de respuestas correctas por grupo del pos-test en los niveles de comprensión de lectura

\begin{tabular}{|c|l|c|c|c|}
\hline \multirow{2}{*}{ Grupo } & Estadísticos & Nivel Literal & Nivel Inferencial & Nivel Crítico Intertextual \\
\hline \multirow{5}{*}{ Experimental } & Media & 3,96 & 4,12 & 4,01 \\
\cline { 2 - 5 } & Muestra & 74 & 74 & 74 \\
\cline { 2 - 5 } & $\begin{array}{l}\text { Desviación } \\
\text { Estándar }\end{array}$ & 0,382 & 0,906 & 0,819 \\
\hline \multirow{5}{*}{ Control } & Media & 3,23 & 2,38 & 1,53 \\
\cline { 2 - 5 } & Muestra & 74 & 74 & 74 \\
\cline { 2 - 5 } & $\begin{array}{l}\text { Desviación } \\
\text { Total }\end{array}$ & 0,803 & 1,069 & 1,263 \\
\hline \multirow{5}{*}{ Estándar } & Media & 3,55 & 3,25 & 2,77 \\
\cline { 2 - 5 } & Muestra & 148 & 148 & 148 \\
\cline { 2 - 5 } & Desviación & 0,703 & 1,319 & 1,638 \\
\hline
\end{tabular}

Para el procesamiento y análisis de la información tanto de la prueba pre test como pos test de los grupos experimental y control, se estableció la escala de evaluación de acuerdo con el número de preguntas formuladas en cada nivel de lectura. Por tanto, el nivel bajo está entre 0 y 2,9; el medio entre 3,0 y 3,9 y el alto entre 4 y 5 . En este sentido, los resultados del grupo control de la Fig. 1 reflejan que para el nivel de desempeño alto, el porcentaje de estudiantes que acertó disminuyó en un $1 \%$, para el nivel de desempeño medio aumentó en un 4\%, y para el nivel de desempeño bajo, el porcentaje de desaciertos a las preguntas formuladas, disminuyó en un 3\%. En el grupo experimental, se observa un notorio aumento en el número de aciertos al finalizar el estudio, en un valor tal, que el desempeño del nivel de comprensión asciende a alto.

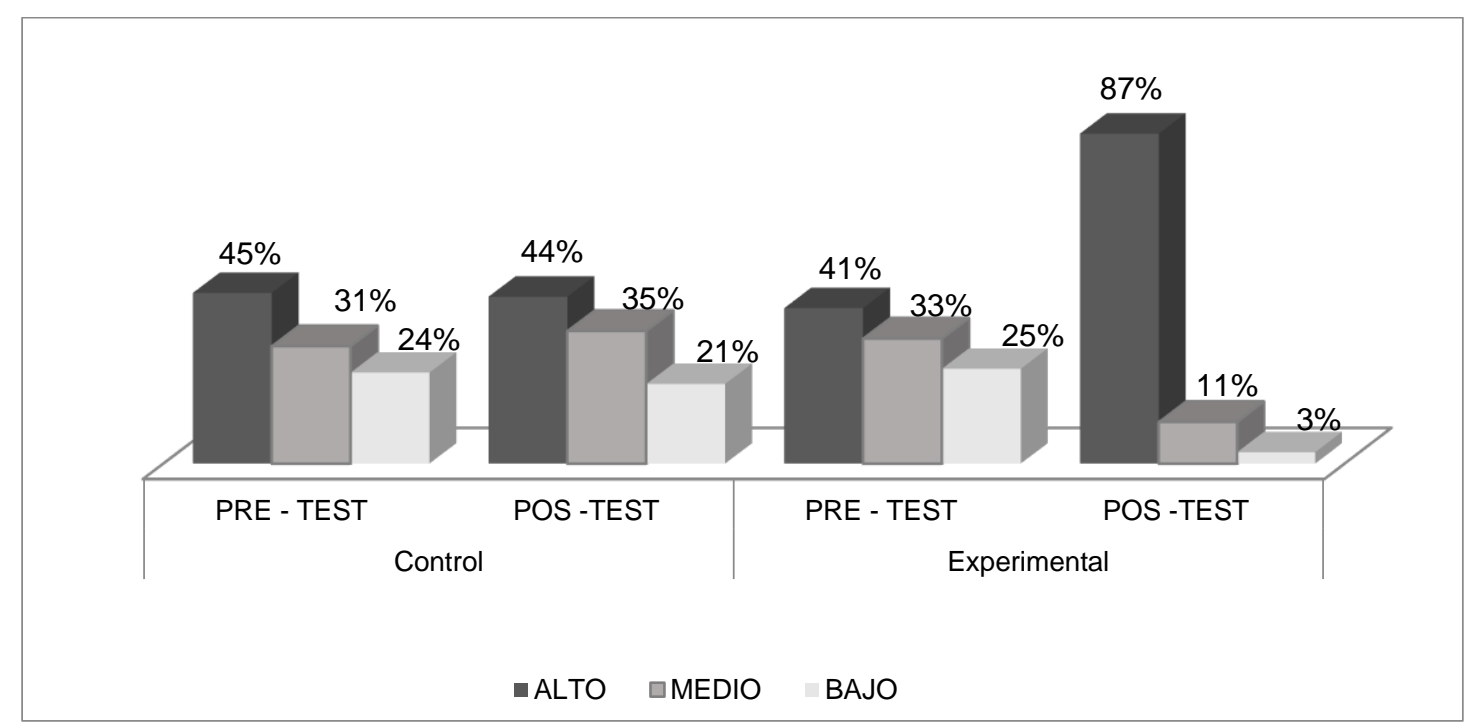

Fig. 1: Desempeño estudiantes nivel literal en pre-test y pos-test

En la Fig.2, se observa que el grupo control presenta una disminución frente al número de preguntas acertadas, ubicando al $56 \%$ de los estudiantes en un nivel de desempeño bajo, En cuanto al grupo experimental, el número de aciertos se incrementa notablemente, en un $62 \%$ para el nivel de desempeño alto. Asimismo, se evidencia que el porcentaje de desaciertos a las preguntas formuladas, disminuye en un $45 \%$. 


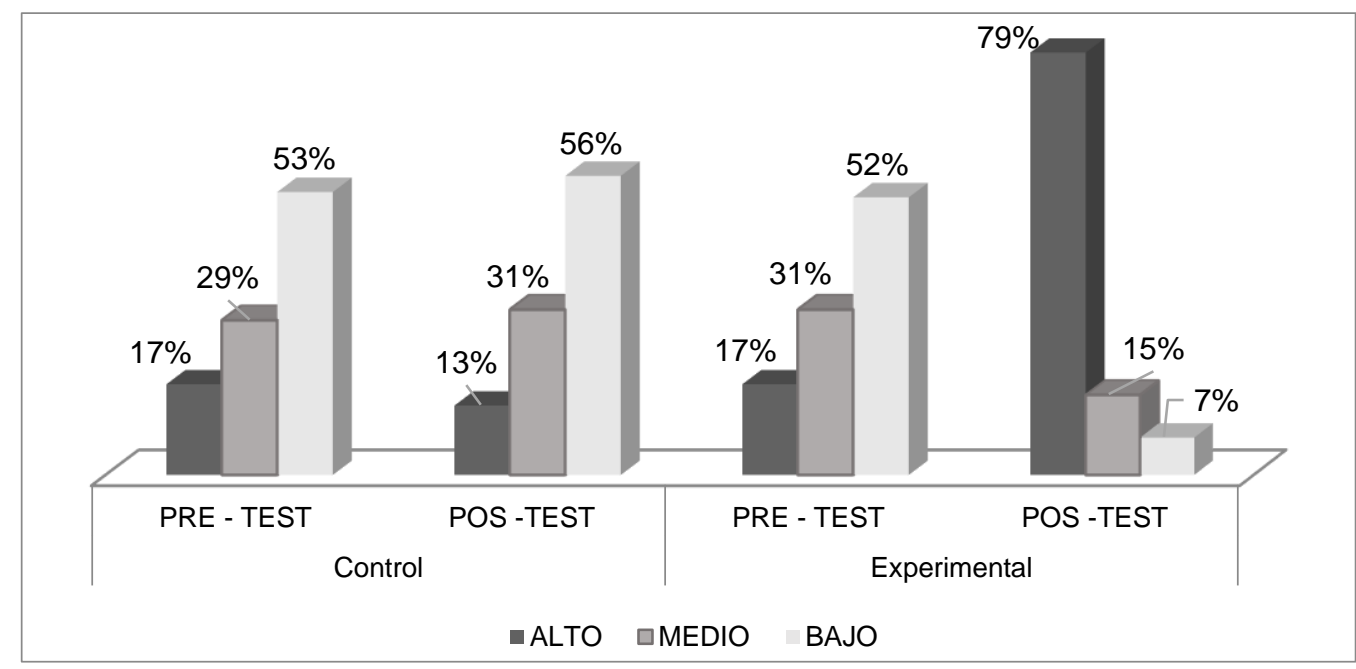

Fig. 2: Desempeño estudiantes nivel inferencial en pre-test y pos-test

En la Fig.3, se observa que los estudiantes del grupo control bajan su desempeño, ubicando al $79 \%$ de ellos en un nivel bajo. Referente al grupo experimental, se presenta un aumento del $60 \%$ de estudiantes que logran posicionarse en el nivel de desempeño alto, asimismo, se evidencia la disminución del $68 \%$ de estudiantes que se ubicaban al inicio del estudio en el nivel de desempeño bajo.

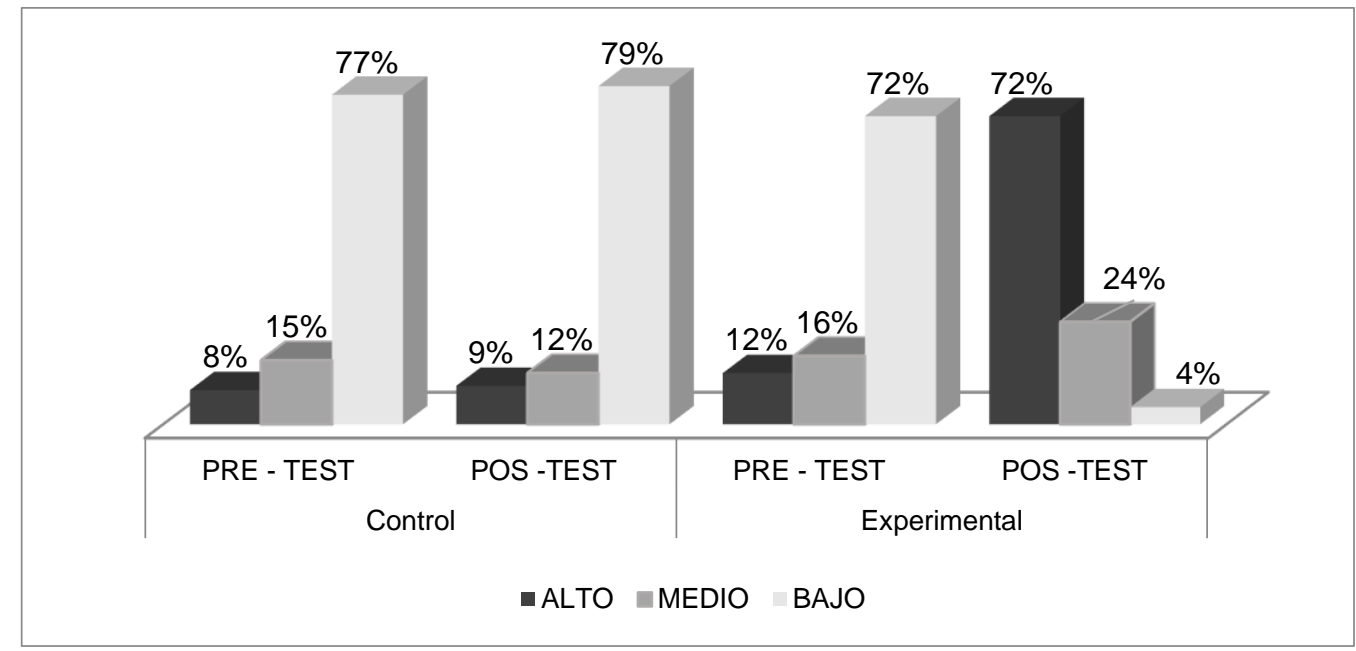

Fig. 3: Desempeño estudiantes nivel crítico intertextual en pre-test y pos-test

\section{Evaluación de los resultados de la aplicación del programa}

Al finalizar el programa, se administró la tercera sección de la escala de estimación a profesores tutores implicados en el programa "Lector-es" para evaluar las dimensiones de medidas y logros, y valoración. En cuanto a la dimensión de medidas y logros, el grado predominante fue alto con un $7 \%$ y muy alto con $93 \%$. Respecto a la dimensión de los resultados del programa estuvo entre alto con un 12 \% y muy alto con un $88 \%$.

\section{Grado de satisfacción de los participantes del programa}

Respecto a la satisfacción de los participantes, el $37,9 \%$ de los estudiantes en formación docente de Licenciatura en Educación Básica les pareció interesante y el 62,1\% muy interesante. En cuanto a los objetivos del programa, el $55,6 \%$ de los estudiantes se encuentran muy satisfechos y el $44,4 \%$ sumamente satisfechos. Relacionado con los contenidos desarrollados, el 38,9\% está muy satisfecho y el $44,4 \%$ sumamente satisfecho. Por último, los recursos y materiales de apoyo que les facilitó el programa han sido adecuados para un $48,3 \%$ y muy adecuados para un $51,7 \%$. De otra parte, el $3,6 \%$ de los estudiantes en formación docente, les ha ayudado algo, al $42,9 \%$ bastante y al 53,6\% mucho a comprender textos del campo académico y profesional para diseñar y aplicación de talleres, guías de aprendizaje, proyectos pedagógicos integradores de áreas, comentarios críticos de obras artísticas; asimismo, a realizar análisis e interpretación de datos estadísticos producto de la aplicación de talleres de comprensión lectora. 


\section{DISCUSIÓN}

De acuerdo con los momentos de evaluación del programa de alfabetización académica "Lector-es", se presenta la discusión del presente estudio investigativo.

\section{Evaluación de las necesidades frente a las prácticas de lectura}

Los estudiantes en formación inicial docente de la Licenciatura en Educación Básica, con énfasis en Matemáticas, Humanidades y Lengua Castellana, en su mayoría leen para actividades propuestas en las asignaturas, para un grupo de estudio y para participar en un evento. Las dos últimas prácticas reflejan cierta motivación por compartir, dialogar lo leído e interés personal, dado que estas no están sujetas a obtener una calificación. Asimismo, leen los materiales elaborados por su profesor y los apuntes propios de su clase, lo que indica que para ellos es más importante lo que suministra su profesor como experto y fuente privilegiada del saber (Cartolari y Carlino, 2011), que los informes de investigación y artículos científicos. También leen páginas web, blogs y documentos digitales. Una explicación a esto es por la modalidad en educación a distancia en que se ofrece la licenciatura. De otra parte, los propósitos de su lectura están relacionados con el control y evaluación de los aprendizajes, leen para cumplir un requisito académico y acreditar saberes. Un grupo de estudiantes en formación docente aún no han vivido una experiencia significativa al interior de las asignaturas. Por esto, es necesario orientar la lectura de los textos académicos en función epistémica para que los estudiantes puedan tener contacto con la producción académica disciplinar y darle un sentido significativo a la lectura (Zarei y Hatami, 2012), y así hacer "uso del lenguaje para pensar, descubrir, transformar, crear y construir el saber" (Serrano Moreno, 2014).

Los docentes de la Licenciatura en Educación Básica, con énfasis en Matemáticas, Humanidades y Lengua Castellana solicitan a sus estudiantes leer de manera frecuente a sus estudiantes, los materiales elaborados por ellos, criterio clave que se valora, pues es una opción que utilizan los docentes para acercar a sus estudiantes a las fuentes escritas (Carlino, 2003). Igualmente, los docentes solicitan leer documentos en soportes electrónicos. Sin embargo, es preciso reflexionar sobre la calidad de esos materiales y la orientación de la lectura en pantalla, por cuanto "leer en línea es mucho más difícil que leer en papel [...] Quizás los chicos sean buenos configurando un ordenador o resolviendo un problema técnico de conexión, pero carecen de actividades estratégicas para leer críticamente en línea" (Cassany, 2011, citado en Corral, 2013, p.141). Por ello, es necesario aplicar una didáctica que permita a los estudiantes aprender a realizar una lectura profunda e interpretativa de este tipo de documentos. De otra parte, en baja frecuencia requiere leer informes de investigación y las publicaciones en revistas. Es de cuestionarse, pues estos documentos son los que direccionan los estudiantes hacia el estudio, comprensión y realización de procesos investigativos en sus áreas de estudio. En cuanto a los propósitos, los docentes solicitan leer a sus estudiantes para exponer y sustentar un tema, lo que indica que este tipo de práctica recae en el estudiante (Carlino 2010), por cuanto es él quien debe leer para exponer frente a sus compañeros. De otra parte, los docentes ofrecen a sus estudiantes apoyo y acompañamiento al inicio y al final de la lectura pero escasos procesos de seguimiento intermedios. Asimismo, orientan la lectura a través de la ejercitación de habilidades, que luego las podrá, aplicarse, a diversas situaciones, y no como una práctica social propia de una cultura disciplinar (Carlino, 2013; Ivanic, 2004; Lea y Street, 1998).

\section{Evaluación inicial del diseño del programa}

El programa transversal de alfabetización académica "Lector-es" es viable para su aplicación, por cuanto responde a las necesidades de los destinatarios, se adecúa a las características del contexto de la modalidad de educación a distancia y a la metodología B-learning y a las características de los estudiantes de formación docente, además favorece la autonomía del estudiante, la participación, la corresponsabilidad y el trabajo en equipo de profesores de lenguaje y de las disciplinas (Carlino, 2010). Para los expertos, la calidad intrínseca del programa es adecuada, dado que es coherente con el contexto y modalidad, ofrece información clara y precisa sobre los aspectos metodológicos. Del mismo modo, los objetivos del programa son suficientes, pertinentes y se adecúan tanto a las necesidades y expectativas de los estudiantes y profesores. Los contenidos del programa son actualizados, relevantes y coherentes con el modelo teórico de alfabetización académica. Las actividades son adecuadas, suficientes y permiten alcanzar los objetivos previstos. Los medios y recursos humanos, tecnológicos y físicos son suficientes y pertinentes. Por último, el sistema de evaluación es suficiente, idónea y permite verificar el logro de los objetivos. Lo anterior, lo concibe Pérez Juste (2014), quien expresa que la evaluación del diseño del programa debe examinar si los elementos del programa están en consonancia con las necesidades a satisfacer, verificar la coherencia interna entre los elementos como objetivos, contenidos, estrategias, actividades, recursos, sistema de evaluación, formadores, etc. y si se ajusta al contexto de aplicación y a las características de los destinatarios. 


\section{Evaluación procesual de la implementación del programa}

Con respecto al nivel inicial de la comprensión lectora de los estudiantes de formación inicial docente de la Licenciatura en Educación Básica del grupo control y experimental, con respecto al nivel literal, se evidencia que ellos pueden identificar la información explicita y clasificarla; reconocer detalles de modo explícito, hacer síntesis de lo leído; asimismo, hacer asociaciones semánticas y relacionar significados de experiencias comunicativas previas con nuevos saberes del texto. Sin embargo, en el nivel inferencial presentan serias dificultades como deducir información implícita, elaborar inferencias, formular hipótesis y nuevas ideas, leer entre líneas. Igualmente, en el nivel crítico intertextual, se les dificulta plantear su propio punto de vista, argumentar, valorar y reflexionar el contenido de la información del texto, asumir una postura crítica ante la opinión de un autor, por último aplicar lo leído a un nuevo contexto, resultados similares obtenidos en la investigación de Durango (2017).

Teniendo en cuenta que la evaluación del desarrollo del programa, trata de analizar si los elementos que constituyen el programa se están desarrollando tal como estaba previsto y dar respuesta a dificultades, obstáculos e imprevistos (Pérez Juste, 2014), el programa "Lector-es" durante su implementación presentó óptimas condiciones para su buen desarrollo, por cuanto en su puesta en marcha, se dirigió a todos los estudiantes de formación docente de los grupos experimentales como se habían previsto, los recursos físicos y tecnológicos previstos se han empleado las secuencias didácticas planeadas se llevaron a la práctica en los periodos de tiempo establecidos con algunos obstáculos en la temporalización, debido a los paros estudiantiles en la universidad. En el marco de la aplicación del programa, los estudiantes en formación docente participaron activamente en el desarrollo de las actividades, igualmente, el profesor del área de lenguaje apoyó a los docentes de las asignaturas para llevar a cabo las secuencias didácticas planificadas, las relaciones cordiales entre docentes y estudiantes y la actitud positiva tanto de estudiantes como profesores favoreció la puesta en marcha del programa. Esto corrobora los resultados obtenidos en estudios investigativos d Moyano (2018), y Moyano y Giudice (2016), con algunos incidentes mínimos en el área de inglés.

\section{Evaluación final de los resultados de la aplicación del programa}

Al realizar la comparación entre pre-test y pos-test, se evidencia que existen diferencias significativas de medias, entre los estudiantes de los grupos experimentales y los de los grupos de control a favor del primero, debido al cambio de porcentaje de los estudiantes del grupo experimental, puesto que en los niveles de desempeño, disminuyeron los porcentajes en los niveles bajos y aumentaron en los niveles más altos. Es así como en el nivel literal, los estudiantes del grupo control persisten con la dificultad de identificar información explícita presentada en el texto, obstaculizando de esta manera, extrapolar sus aprendizajes a los niveles superiores de comprensión lectora. En el caso del grupo experimental, se observa un notorio aumento en el número de aciertos al finalizar el estudio, en un valor tal, que el desempeño del nivel de comprensión asciende a alto. Referente al nivel inferencial, el grupo control presenta un desempeño muy bajo, lo que refleja la incapacidad de los estudiantes en deducir nuevas hipótesis a partir de la información implícita en el texto. En cuanto al grupo experimental, se incrementó notablemente a un nivel de desempeño alto, lo que demuestra que el programa "Lector-es" ayudó a los estudiantes a fortalecer la habilidad para extraer conclusiones y conjeturas con base en la información presentada. Respecto al nivel crítico intertextual, los estudiantes del grupo control bajan su desempeño, ubicándose en un nivel bajo, demostrando de esta manera, una acentuada problemática a la hora de asumir posición y transferir lo leído, mientras que el grupo experimental presentó un aumento logrando posicionarse en el nivel de desempeño alto. Por lo anterior, se concluye que el programa "Lector-es" es eficaz, por cuanto se logró mejorar el nivel comprensión lectora de los estudiantes de formación docente.

El programa transversal de alfabetización académica "Lector-es" es eficiente, dado que existen los medios, los espacios, el tiempo y los recursos humanos, físicos y tecnológicos para su aplicación (Pérez Juste, 2014). Del mismo modo, el programa utiliza técnicas e instrumentos para decidir la eficacia del programa respecto a las características de los contenidos y objetivos que se desearon evaluar. De otra parte, se planificaron pruebas y demás instrumentos para la recolección de información, en el momento de diseñar el programa, a fin de asegurar la máxima coherencia con los objetivos. Los recursos (humanos, materiales, organizativos y espacio temporales) requeridos son acordes con los resultados conseguidos; asimismo, el programa recogió información sobre el grado de satisfacción de los participantes y de la evaluación inicial, procesual y final para elaborar los resultados.

Para los estudiantes en formación docente de la Licenciatura en Educación Básica de la FESAD fue satisfactorio participar en el programa transversal de alfabetización académica "Lector-es", dado que les pareció interesante las actividades, los contenidos, y los recursos. De igual manera, les ayudó a manejar los géneros discursivos del área disciplinar (Parodi, et al., 2010), con el fin de asimilar y transformar el 
conocimiento de las asignaturas de "Fundamentos y didácticas de las artes plásticas", "Fundamentos y Didácticas de la Educación Física", "Lengua Castellana para la Educación Básica Primaria", "Profundización en el área del Inglés para la Educación Básica" y Profundización en el área de las matemáticas para la Educación Básica Igualmente, a leer, valorar y apreciar obras artísticas, a leer e interpretar tablas y gráficas estadísticas de estudios investigativos, a leer y comprender géneros académicos y profesionales propios de formación disciplinar docente. Por otro lado, el programa les ayudó a diseñar talleres de comprensión lectora, talleres para la enseñanza del inglés y proyectos pedagógicos integradores de áreas, lo que conllevó a la participación en actividades propias de su formación docente (Carlino, 2013). Finalmente, reconocen el valor a la lectura como proceso de su formación universitaria enmarcada en contextos sociales (Jerez-Rodríguez y Navas-Ríos, 2019).

El proceso investigativo se llevó a cabo en las siguientes fases: 1) construcción teórica, conceptual y metodológica de la investigación; 2) Identificación de las necesidades de las prácticas de lectura en los estudiantes y las prácticas de enseñanza de la misma; 3) la evaluación inicial del diseño del programa de alfabetización académica "Lector-es"; 3) Evaluación procesual de la implementación del programa; 4) Evaluación final de los resultados de la aplicación del programa "Lector-es". Vale la pena resaltar que la anterior evaluación rigurosa, avala la utilidad del Programa Transversal de Alfabetización Académica "Lectores" como opción pedagógica para fomentar la enseñanza de la lectura como eje trasversal del plan de estudios, apropiación de los contenidos de las asignaturas del área disciplinar y de profundización, y la participación de actividades propias de un docente de Educación Básica.

\section{CONCLUSIONES}

Del anterior estudio investigativo se concluye: 1) El programa transversal de alfabetización académica "Lectores" resulta efectivo para la enseñanza de la lectura, apropiación de los contenidos de las asignaturas del área disciplinar y de profundización, y la participación en actividades propias de una cultura académica y profesional, en este caso en la formación docente. 2) El programa "Lector-es", se apoya en los principios de la lectura como función epistémica y sociocultural, y como actividad estratégica para aprender en contexto las asignaturas de "Fundamentos y Didácticas de la Educación Física", "Lengua Castellana para la Educación Básica Primaria", "Profundización en el área del Inglés para la Educación Básica" y Profundización en el área de las matemáticas para la Educación Básica. 3) La aplicación del programa "Lector-es" implica un trabajo interdisciplinario entre, directivos, profesores especializados de lenguaje y docentes del área disciplinar y de profundización y estudiantes.

\section{REFERENCIAS}

Acuerdo 050 de 2009, por el cual se establecen los criterios para la implementación del Sistema de Créditos y se definen las Áreas de Estructuración Curricular de los Programas de Pregrado, en la Universidad Pedagógica y Tecnológica de Colombia, Tunja, Colombia (2008)

Bazerman, Ch., J. Little y otros cuatro autores, Reference Guide to Writing Across the Curriculum, 1르. Ed., 5-20, ISBN: 1932559-44-2, West Lafayette, Indiana (2005)

Biggs, J., What the Student Does: Teaching for Enhanced Learning, Higher Education Research \& Development, doi: 10.1080/0729436990180105, 18 (1), 57-75 (1999)

Bogel, F. y K. Hjortshoj, Composition Theory and the Curriculum. In F. Bogel y K. Gottschalk (Eds.), Teaching Prose. A guide for writing instructors, (pp. 1-19), Norton, New York, United States (1984)

Cabero, J. y M. D. Llorente, Del eLearning al Blended Learning: Nuevas acciones educativas, Quaderns Digitals / Quaderns, ISSN-e 1575-9393 (51), 1-9 (2008)

Calle Arango. L., Educación Superior: La Alfabetización en Géneros Discursivos, 10.1590/2175-623665114, Educação \& Realidade, 43(2), 355-381 (2018)

Carlino, P. Alfabetización académica: Un cambio necesario, algunas alternativas posibles. Educere, ISSN: 1316-4910, 6 (20), 409-420 (2003)

Carlino, P., Alfabetización Académica Diez años Después, Revista Mexicana de Investigación Educativa, ISSN: 14056666, 18(57), 355-381 (2013)

Carlino, P., Dos variantes de la Alfabetización Académica cuando se Entrelazan la Lectura y la Escritura, doi: 10.11144/Javeriana.syp36-71.dvaa, Signos y Pensamiento, 36(71), 16-32 (2018)

Carlino, P., Reading and Writing in the Social Sciences in Argentine universities. In Ch. Bazerman., R. Krut y otros tres autores (Eds.), Traditions of Writing Research (pp. 283-296), Routledge / Taylor and Francis, Oxford, United Kingdom (2010)

Cartolari, M. y P. Carlino, Leer y tomar apuntes para aprender en la formación docente: Un Estudio Exploratorio, Revista Internacional de Investigación en Educación, ISSN 2027-1174 4(7), 67-86 (2011) 
Cartolari, M., P. Carlino y L. Colombo, Reading and Note Taking in Monological and Dialogical Classes in the Social Sciences, doi: 10.14221/ajte.2013v38n6.7, Australian Journal of Teacher Education, 38(6) ,158-183 (2013)

Castelló, M., Los retos actuales de la alfabetización académica: estado de la cuestión y últimas investigaciones, doi.org/10.14483/10.14483/udistrital.jour.enunc.2014.2.a13, Enunciación, 19(2), 346-365 (2014)

Chalmers, D. y R. Fuller, Teaching for Learning at University, 1ㅡㄹ., 52-122, ISBN: 978-0749420413, Kogan Page, London (1996)

Colombo, L. y M. Prior, How does Faculty Conceptions on Reading, Writing and their Role in the Teaching of Academic Literacies Influence their Inclusive Attitude, doi: 10.5007/2175-8026.2016v69n3p115prácticas, Ilha do Desterro, 69(3), 115-124 (2016)

Corral Cañas, C., Leer y escribir en la red, de Daniel Cassany Celia Corral Cañas. Caracteres. Estudios culturales y críticos de la esfera digital, ISSN: 2254-4496 2(1), 139- 143(2013)

Durango, Z., Niveles de Comprensión Lectora en los Estudiantes de la Corporación Universitaria Rafael Núñez (Cartagena de Indias), ISSN: 0124-5821, Revista Virtual Universidad Católica del Norte, 51, 156-174 (2017)

González, B. y V. Vega, Lectura y Escritura en la Educación Colombiana: Herencia y Desconstrucción, doi: 10.26564/16926250.655, Revista Interacción, 12, 195-201 (2013)

González, B.Y., A. A. Salazar Sierra y otros dos autores, Acciones para la construcción de una política de Lectura y Escritura universitaria, Folios, ISSN 0123-4870, (41), 143-155 (2015)

Jerez-Rodríguez, S. y M. E. Navas-Ríos, Leer, Escribir y Comunicarse en Otro Idioma con Nuevas Prácticas Letradas Fuera del Aula de Clase, doi.org/10.4067/S0718-07642019000200315, Inf. Tecnol., 30(2), 315-326 (2019)

Lea, M. y B. V. Street, Student writing in Higher Education: An academic Literacies Approach, doi: 10.1080/03075079812331380364, Studiesin Higher Education, 23, 157-172, Kluwer Academic Pub., London (1998)

López-Bonilla, G., Prácticas Disciplinares, Prácticas Escolares: Qué son las Disciplinas Académicas y Cómo se Relacionan con la Educación Formal en las Ciencias y en las Humanidades, Revista mexicana de investigación educativa, ISSN: 1405-6666, 18(57), 383-412 (2013)

Marinkovich, J., M. Velásquez, A. Córdova y C. Cid, Academic Literacy and Genres in University Learning Communities, doi: 10.5007/2175-8026.2016v69n3p95, Ilha do Desterro, 69(3), 95-113 (2016)

MEN, Ministerio de Educación Nacional, Colombia, Lineamientos de Calidad para las Licenciaturas de Educación (Programas de Formación Inicial de Maestros), Bogotá (2014)

Mora Venegas, P., Prácticas Docentes y Motivación por la Lectura, Un Estudio Empírico desde la Didáctica de la Lengua, Tesis Doctoral, Univ. Internacional Iberoamericana, México (2017)

Moyano, E. I., La Enseñanza de la Lectura y la Escritura Académicas mediante Programas a lo largo del Curriculum Universitario: Opción teórica, didáctica y de gestión, doi: 10.1590/0102-445074896274115057, Delta: Documentação de Estudos em Lingüística Teórica e Aplicada, 34(1), 235-267 (2018)

Moyano, E. y J. Giudice, Un Programa de Lectura y Escritura Universitario: Lineamientos teóricos, características y resultados de aplicación, doi: 10.26564/16926250.655, Revista Grafía- Cuaderno de trabajo de los Profesores de la Facultad de Ciencias Humanas, Universidad Autónoma de Colombia, 13(1), 33-59 (2016)

Newell, G. y Winograd, P, The Effects of Writing on Learning from Expository Text, doi: 10.1177/0741088394011003002, Written Communication, 6, 196-217 (1989)

Parodi, G., R. Ibáñez R., R. Vanegas y C. González, Identificación de Géneros Académicos y Géneros Profesionales: Principios teóricos y propuesta metodológica, en Giovanni Parodi (ed.), Alfabetización académica en el siglo XXI: leer y escribir desde las disciplinas, (pp. 249-290) Planeta, Santiago (2010)

Pérez Juste, R., Evaluación de Programas Educativos, 2ª ED., 213-257, ISBN: 978-84-7133-755-9, La Muralla, Madrid (2014)

Pérez, M. y G. Rincón, (Coords.) ¿Para qué se Lee y se Escribe en la Universidad Colombiana? Un aporte a la consolidación de la cultura académica del país, 1르 Ed., 297-306, ISBN: 978-958-716-628-6, Pontificia Universidad Javeriana, Bogotá (2013)

Poblete, S. E., y B. E. Figueroa y M. S. Aillon, Percepciones sobre la Aplicación de un Diseño Multimodal para la Alfabetización Científica en el Área de la Salud, doi.org/10.4067/S0718-07642017000400021, Inf. Tecnol., 28(4), 191-202 (2017)

Radloff, A., Communication-in-context. A Commonwealth Staff Development Funded Project, Perth: Curtin University of Technology (1988)

Russell, D., Contradictions Regarding Teaching and Writing or Writing to learn in the disciplines:What we have learned in the USA, Revista de Docencia Universitaria, ISSN 1887-4592, 11(1), 161-181 (2013)

Salazar, R. y Melo, A., Lineamientos Conceptuales de la Modalidad de Educación a Distancia. En N. Arboleda y C. Rama (Eds.), La educación superior a distancia y virtual en Colombia: nuevas realidades (pp. 81-112). Colombia: ASESAD (2013) 
Salazar-Sierra, A., O. Sevilla-Rengifo y otros ocho autores, Lectura y Escritura en la Universidad: Contribución para Reconstruir una Historia, doi: 10.11144/Javeriana.m8-16.leuc, Magis, Revista internacional de investigación en educación, 8(16), 51-70 (2015)

Sánchez Upegui, A., Alfabetización Académica: Leer y Escribir desde las Disciplinas y la Investigación, doi: 10.22507/rli.v13n2a18, Revista Lasallista de Investigación, 13(2), 200-209 (2016)

Santos, D., La lectura y Escritura Académicas y la Vida Política en el Aula Universitaria: Hacia una Literacidad Ética, doi: 10.15446/fyf.v29n2.60193, Forma y Función, 29(2), 157-181 (2016)

Serrano, S., La Lectura, la Escritura y el Pensamiento. Una Función Epistémica e Implicaciones Pedagógicas. Lenguaje, ISSN 0120-3479, 42(1), 97-124 (2014)

Skillen, J., M. Merten, N. Trivett, y A. Percy, The Ideal Approach to Learning Development: A Model for Fostering Improved Literacy and Learning Outcomes for Students, Annual Meeting of the Australian Association for Research in Education, 03-20, Auastralia, December (1998)

Solé, I., Competencia Lectora y Aprendizaje, Revista Iberoamérica de educación, ISSN 1022-6508, (59), 43-61 (2012)

Waigandt, D., A.M. Noceti y L.M. Zapata, Engineering the Future: Teaching Reading and Writing at the Universidad Nacional de Entre Rios, doi: 10.5007/2175-8026.2016v269n3p173, Ilha do Desterro, 69(3), 173-187 (2016)

Yubero, S. y E. Larrañaga, Lectura y Universidad: Hábitos Lectores de los Estudiantes Universitarios de España y Portugal, doi: 10.3145/epi.2015.nov.03, El profesional de la información, 24(6), 717-723 (2015)

Zarei, A. y G. Hatami, On the Relationship between Self-regulated Learning Components and L2 Vocabulary Knowledge and Reading Comprehension, doi: 10.4304/tpls.2.9.1939-1944G, Theory and Practice in Language Studies, 2(9), 1939$1944(2012)$ 